\title{
Development of a Fatty Liver Model by Restricted Feeding of Lactating Sheep
}

\author{
Wei Yang ${ }^{1}$, Xiaobing Li' ${ }^{2}$, Guowen Liư', Hongyou Zhang ${ }^{1}$, Yuanyuan Chen ${ }^{1}$, Cheng Xia' \& Chuang Xu'
}

\begin{abstract}
Background: As a frequent subclinical disease, fatty liver disease (FLD) is associated with a severe negative energy balance (NEB) during the early lactation period, and usually cause of economic loss to dairy farmers. Liver biopsy is the gold standard for the assessment of FLD. However, as an invasive procedure, liver biopsy has several limitations and such procedures are not readily available to dairy farmers. To further evaluate FLD in dairy cows, a FLD model of lactating sheep was developed by simulation of the state of negative energy balance (NEB).

Materials, Methods \& Results: Fourteen pregnancy thin-tail ewes were divided into control group (CG, $n=4)$, non-lamb restrained feeding group (NRG, $\mathrm{n}=4$ ) and single birth restrained feeding group $(\mathrm{SRG}, \mathrm{n}=6)$. After lambing, $\mathrm{NRG}$ and SRG ewe were received a feed restrained diet for 16 days. Liver biopsies and blood was collected on days 1, 4, 7, 10, 13, and 16, and biochemical parameters were analyzed. With restricted feeding and lactation administration, ewes in SRG showed increased liver fat concentrations (LFC) from days 4 post-administration and severe LFC was detected at day 13. Compared with CG, SRG sheep showed significant lower concentration of serum glucose (Glu) from days 7-13 and higher non-esterified fatty acid (NEFA) from days 4-16, $\beta$-hydroxybutyric acid (BHBA) from days 4-16, triglyceride from days 4-16, low-density lipoprotein cholesterol from days 4-16, lactate dehydrogenase (LDH) from days 13-16, aspartate aminotransferase (AST) at days 16. While, ewes in NRG showed normal LFC levels, and high concentration of serum Glu and insulin from days 4-16 were detected than CG and SRG ewes. With restricted feeding, ewes in NRG and SRG showed significant low level of revised quantitative insulin sensitivity check index from days 4-16 and high level of liver total cholesterol (TC) at day 16. Liver pathological characteristics showed LFC of NEB sheep was first detected around the liver portal area.

Discussion: In this study, a model of FLD in lactating thin-tail sheep was developed by restricted feeding. Serum glucose concentrations were sharply decreased in SRG sheep that was due to the large energy requirements for lactation and low energy provided by a restricted diet. While non-lactating NRG sheep demonstrated lower fat mobilization, which was considered to contribute to the high concentrations of serum glucose, as compared to SRG sheep. Meanwhile, in a state of NEB, oxaloacetic acid, which is generated by glycolysis and glycogenic amino acids, tends to be used for gluconeogenesis, that a generous amount of NEFA is incompletely oxidized to generate ketone body in SRG sheep, which is a major component of BHBA. Liver TC concentrations were significantly higher in NRG sheep than those in the SRG sheep, while liver triglyceride was significantly lower. The high level of liver TC in NRG sheep was considered to induce removal of triglyceride from the liver in the form of VLDL. Compared with CG sheep, although higher levels of liver TC were detected in SRG sheep on postpartum day 16, these levels were considered too low to induce significant depletion of triglycerides from the liver. In this study, the increase in serum AST and LDH was considered to cause by oxidative stress in mitochondria, and LDH concentrations was considered more sensitively than AST for LFC caused by NEB. Liver pathological characteristics showed that FLD caused by NEB had a major impact on reduced LFC, although no significant liver fibrosis was detected. While different from FLD caused by high-fat diet, TG was first accumulates around the hepatic lobules and LFC of NEB sheep was first detected around the liver portal area. It was considered that high concentrations of NEFA are prioritized for oxygenation in the liver portal area, which results in triglyceride accumulation.
\end{abstract}

Keywords: fatty liver, sheep, negative energy balance, non-esterified fatty acid. 


\section{INTRODUCTION}

Fatty liver disease (FLD) is one of the most common disorders in dairy cows, especially among high milk-producing cattle, and affects up to $50 \%$ within the first few weeks postpartum [14]. Furthermore, FLD of dairy cows is associated with a severe negative energy balance (NEB) during the early lactation period as a consequence of insufficient energy intake to sustain the high energy requirements for milk production [7]. To counteract a NEB, fat from adipose tissue stores in dairy cows was mobilized to form glycerol for gluconeogenesis, while large quantities of non-esterified fatty acid (NEFA) in the blood are taken up by the liver and oxidized to ketone bodies or esterified to triacylglycerol, which leads to ketosis and FLD $[12,17,18]$. FLD usually causes a decrease milk yield and several secondary diseases including displaced abomasum and reproductive disorders, thus lead to the major economic losses $[5,10]$. Hence, further evaluations of FLD pathogenesis are necessary to reduce the risk of progressive liver diseases. Normally, liver biopsy is the gold standard for the assessment of FLD due to clinical symptoms and blood parameters were insufficient for specific diagnosis of FLD [3]. However, as an invasive procedure, liver biopsy has several limitations like unavailable procedures for dairy farmers and in vivo research. Therefore, a proper animal model is a prerequisite for further investigations. In this research, a FLD model of parturition sheep was developed by simulation of the state of NEB. The clinical status and characteristics of glycolipid metabolism were also analyzed.

\section{MATERIALS AND METHODS}

\section{Diet and feeding}

Fourteen pregnancy thin-tail ewes with parities of 2-3 were acquired. After lambing, the ewes were fasted for $12 \mathrm{~h}$ and allowed to nurse for lamb freedom and were then allocated to three experimental groups: control group ( $\mathrm{CG}, \mathrm{n}=4)$, each gave birth to a single lamb that remained with the ewe in individual pens and the ewe was received a normal diet of $2 \mathrm{~kg}$ peanut hay and $300 \mathrm{~g}$ concentrate (Contains $2.1 \mathrm{~kg}$ of Dry matter/d, 12.8 MJ of NEL/d) feed twice a day; nonlamb restrained feeding group (NRG, $n=4)$, each gave birth to a single lamb that was isolated with the ewe after 24-h of suckling colostrum (the ewes were not artificially milked) and the ewe was received a feed restrained diet that consisted of $400 \mathrm{~g}$ of peanut hay and $60 \mathrm{~g}$ of concentrate (Contains $0.4 \mathrm{~kg}$ of Dry matter/d, 2.6 MJ of NEL/d) feed twice a day; single birth restrained feeding group $(\mathrm{SRG}, \mathrm{n}=6)$, each gave birth to a single lamb that remained with the ewe for freedom suckling and the ewe was received the same diet as the NRG. Ewes in all groups were fed with the designated diet for 16 days.

Body weight measurement, blood and liver biopsy collection

Body weight (BW) of each sheep was measured before morning feeding on days 1 (12 $\mathrm{h}$ postpartum), 4, 7, 10, 13, and 16 using a digital scale. At these times, blood was collected by jugular vein puncture, and the serum was subsequently stored at $-80^{\circ} \mathrm{C}$. Then liver biopsies were collected through a $0.5-\mathrm{cm}$ rightside incision between the 11th and 12th rib from the mid-scapula to tuber coxae using a biopsy paracentesis needle (Cone TZ14-16) ${ }^{1}$ for triglyceride analysis.

\section{Liver and bile collection}

On the final day of the experimental period (postpartum day 16), each sheep was anesthetized by intramuscular injection of ketamine hydrochloride (50 $\mathrm{mg} / \mathrm{kg} \mathrm{BW})$ and xylazine hydrochloride $(50 \mathrm{mg} / \mathrm{kg}$ BW) and euthanized by exsanguination. After exsanguination, the liver was obtained, and part of the liver was frozen in liquid nitrogen for Oil Red $\mathrm{O}$ staining. The remaining tissue was fixed in $4 \%$ formaldehyde for hematoxylin/eosin $(\mathrm{H} \& \mathrm{E})$ and picrosirius red staining. Bile was collected from the gallbladder and stored at $-80^{\circ} \mathrm{C}$ for further analysis.

\section{Analysis of biochemical parameters}

Blood biochemical parameters, including serum glucose (Glu), NEFA, $\beta$-hydroxybutyric acid (BHBA), triglyceride, total cholesterol (TC), lowdensity lipoprotein cholesterol (LDL-C), aspartate aminotransferase (ALT), aspartate aminotransferase (AST), alkaline phosphatase (ALP), cholinesterase (CHE), lactate dehydrogenase ( $\mathrm{LDH})$, and bile TC and total bile acids (TBA) were analyzed using a biochemical kit ${ }^{2}$ using an automatic clinical analyzer (Synchron DXC800) ${ }^{3}$. Serum insulin was analyzed using an enzyme-linked immunosorbent kit ${ }^{4}$. The revised quantitative insulin sensitivity check index (RQUICKI) was calculated as described by Holtenius and Holtenius [9]. Liver triglyceride, glycogen and TC were analyzed using a kit ${ }^{4}$. 
Statistical analysis

All data were analyzed using SPSS statistical software (version 19.0) and the results are presented as means \pm SD. Differences in BW, blood biochemical parameters, and liver triglyceride levels were identified by repeated measures analysis of variance. Glycogen and TC levels in bile and liver were analyzed using the post-hoc Duncan's multiple range tests. $P<0.05$ was presented statistically significant.

\section{RESULTS}

\section{Clinical symptoms and liver biopsies}

The BW of ewes in the SRG was dramatically lower than CG from postpartum day 10 (Figure 1), and there was no significant difference between SRG and NRG during the experimental period. As show in Figure 1, hepatic triglyceride levels were significantly increased in the SRG than the CG and NRG, and there was no significant difference in values between the $\mathrm{CG}$ and NRG sheep.
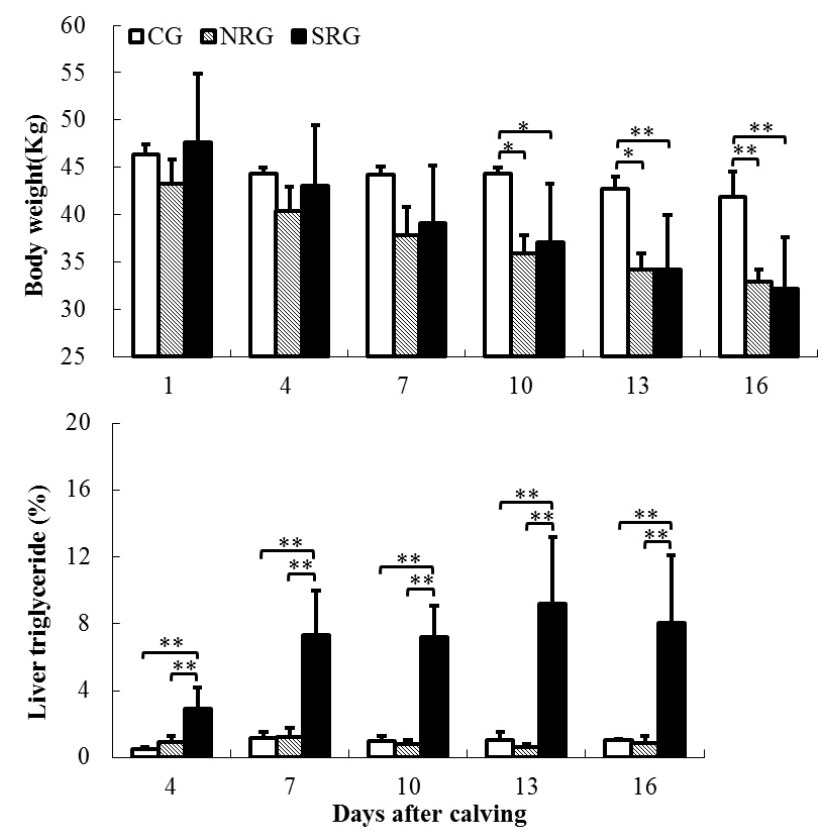

Figure 1. Body weight and liver triglyceride on postpartum days 1, 4, 7, $10,13$, and 16 in the CG, NRG, and SRG. [ $P<0.05 \geq 0.01 ; * * P<0.01]$.

\section{Biochemical parameters of plasma, liver and bile}

As shown in Figure 2, CG sheep had stable serum glucose concentration during the entire experimental procedure, and there were no significant differences in the NRG and CG sheep during the experimental period, except for postpartum days 7 and $13(P=0.010$ and 0.039 , respectively). Compared with $\mathrm{CG}$, serum glucose concentration in SRG sheep was significantly lower on postpartum days 7 and $10(P=0.040$ and 0.046 , respectively). Serum NEFA concentration in the SRG sheep was significantly higher than in the CG and NRG sheep after postpartum day 4 and serum BHBA in SRG sheep sharply increased during initial lactation stage and these high concentrations were maintained during the experimental period. NEFA concentrations in NRG sheep were only significantly higher than in the CG sheep on postpartum days 7 and $10(P=0.025$ and 0.026 , respectively), while there were no significant differences in BHBA concentrations between ewes in the NRG and CG during the entire experimental period.
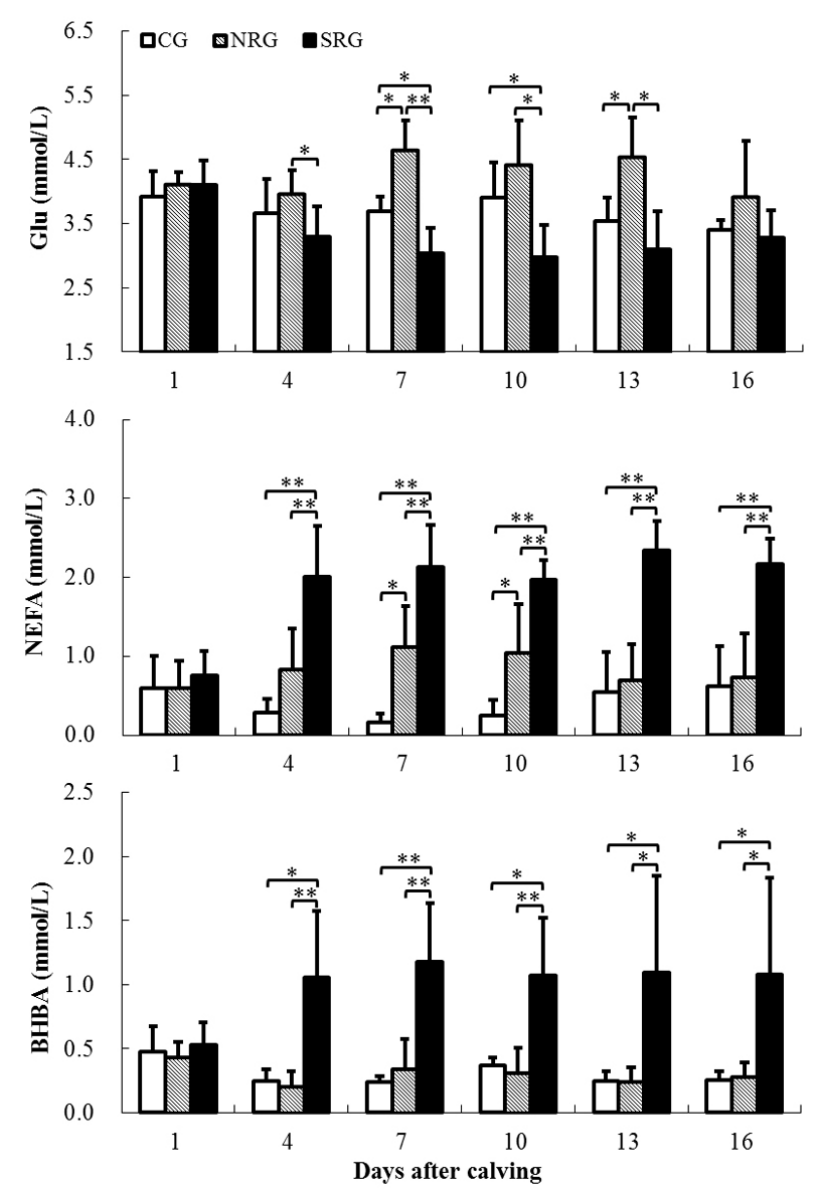

Figure 2. Serum glucose, non-esterified fatty acid (NEFA), and $\beta$-hydroxybutyric acid (BHBA) concentrations at postpartum days 1, 4, 7, 10, 13 , and 16 days in the CG, NRG, and SRG. $[* P<0.05 \geq 0.01 ; * * P<0.01]$.

As shown in Figure 3, serum triglyceride concentrations in SRG sheep sharply increased during the early period of lactation and were significantly higher than in CG sheep during the experimental period. Serum triglyceride concentrations were higher in NRG sheep than in CG sheep, but there was no significant 
difference detected between these two groups. Serum LDL-C concentrations were high in SRG compared with the $\mathrm{CG}$ and a significant difference between NRG and $\mathrm{CG}$ was detected on postpartum day $7(P=0.012)$, while there were no significant differences between NRG and SRG, except for postpartum day $16(P=$ 0.017). Serum TC concentrations in the restricted feed groups were higher than those in the $\mathrm{CG}$, while there was no significant difference among the three groups.

As shown in Figure 4, Serum AST concentrations in SRG sheep were significantly higher than in CG sheep on postpartum day $16(P=0.026)$. Serum LDH concentrations in the SRG sheep were significantly higher than in other groups on postpartum day 13 and 16. There were no significant differences in serum concentrations of ALT, CHE, and ALP among the three groups (data not shown).

In the present study, serum insulin and RQUICKI were also analyzed. As shown in Figure 5, although serum insulin concentrations in SRG sheep were higher than those in the CG, there was no significant difference detected. The RQUICKI values in CG sheep were significantly higher than those in other groups after postpartum day 4 . There were no signifi-
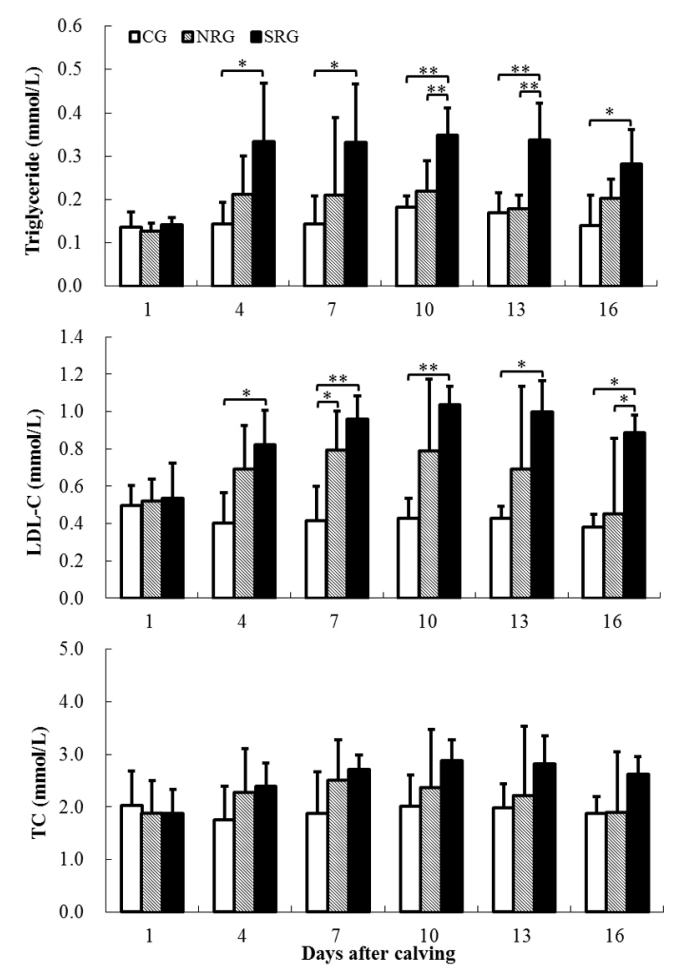

Figure 3. Serum triglyceride, low-density lipoprotein (LDL-C), and total cholesterol (TC) concentrations at postpartum days 1, 4, 7, 10, 13, and 16 days in the CG, NRG, and SRG. [ ${ }^{*} P<0.05 \geq 0.01 ; * * P<0.01$ ]. cant differences in RQUICKI values among sheep in the two restricted feeding groups.

Biochemical parameters of the liver and bile were only analyzed using samples obtained on postpartum day 16. As shown in Table 1, there were no significant differences in hepatic glycogen and bile TC and TBA concentrations among the three groups. TC levels in the livers of NRG sheep were higher than in other groups, while levels in the SRG sheep were significantly higher than in CG sheep.

\section{Pathological changes in the liver}

As shown in Figure 6, livers from the CG and NRG sheep demonstrated normal hepatocytic texture with a radiated hepatic cord and sinusoids around the hepatic lobule. While liver histopathological analyses of SRG sheep showed differences in the LFCs, those of NEB sheep tended to accumulate around the portal area. Oil red $\mathrm{O}$ staining of FLD can clearly display the tendency of LFC spread from the liver portal area to the hepatic lobule. In this study, picrosirius red was employed to assess the degree of liver fibrosis and steatohepatitis, which revealed no significant fibrosis among the four groups [13].

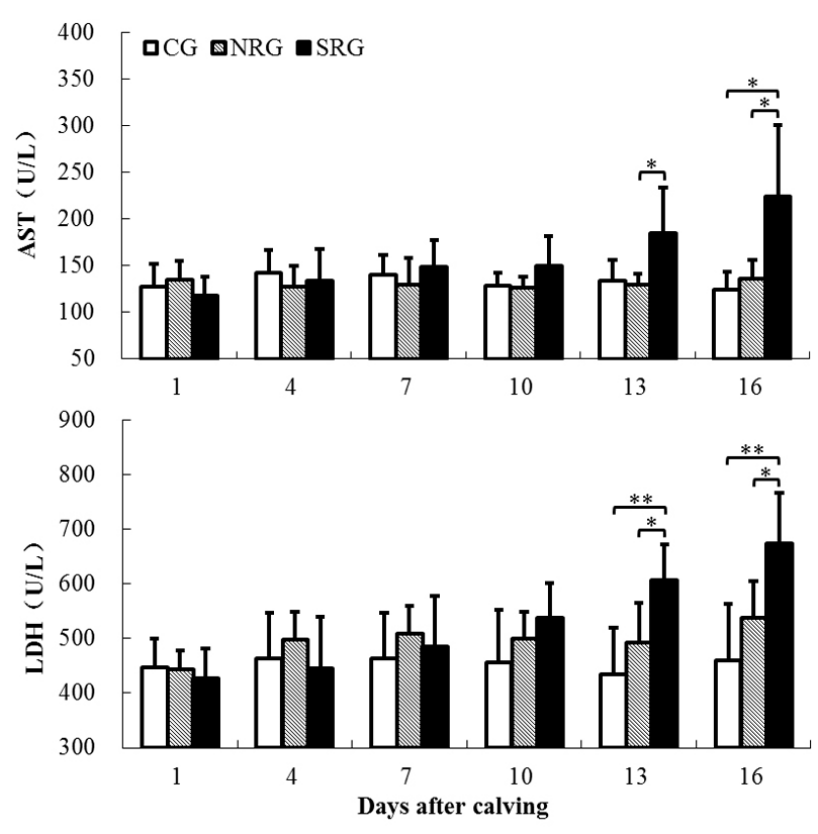

Figure 4. Serum aspartate aminotransferase (AST) and lactate dehydrogenase $(\mathrm{LDH})$ concentrations at postpartum days $1,4,7,10,13$, and 16 days of CG, NRG, and SRG. [ $\left.{ }^{*} P<0.05 \geq 0.01 ; * P<0.01\right]$. 


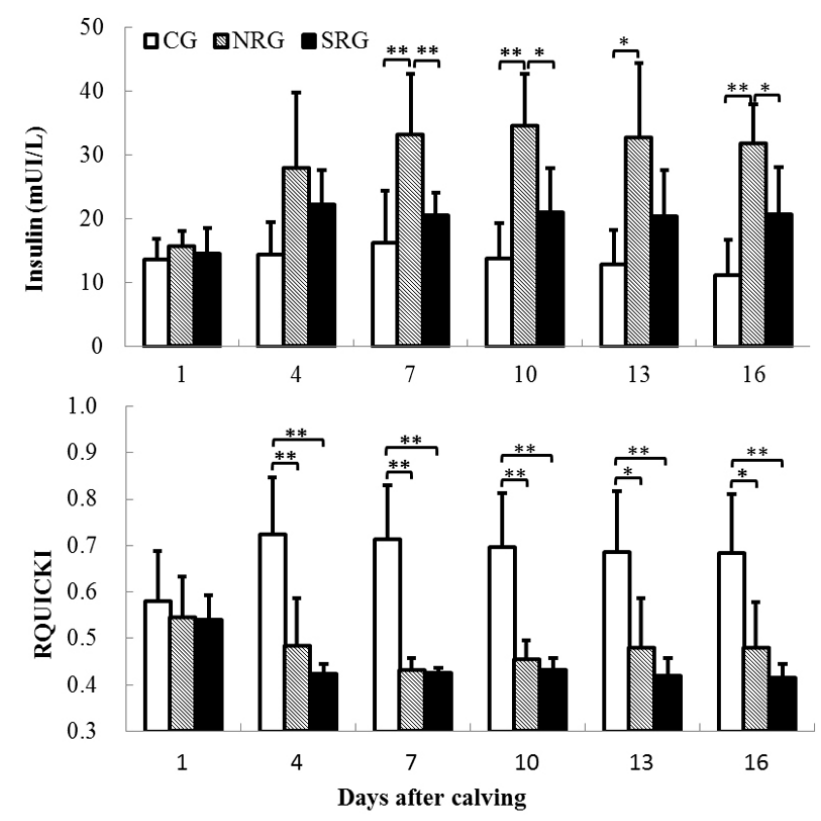

Figure 5. Serum insulin and revised quantitative insulin sensitivity check index (RQUICKI) aspartate aminotransferase levels at postpartum days 1,4 , $7,10,13$, and 16 days in CG, NRG, and SRG. $[* P<0.05 \geq 0.01 ; * * P<0.01]$
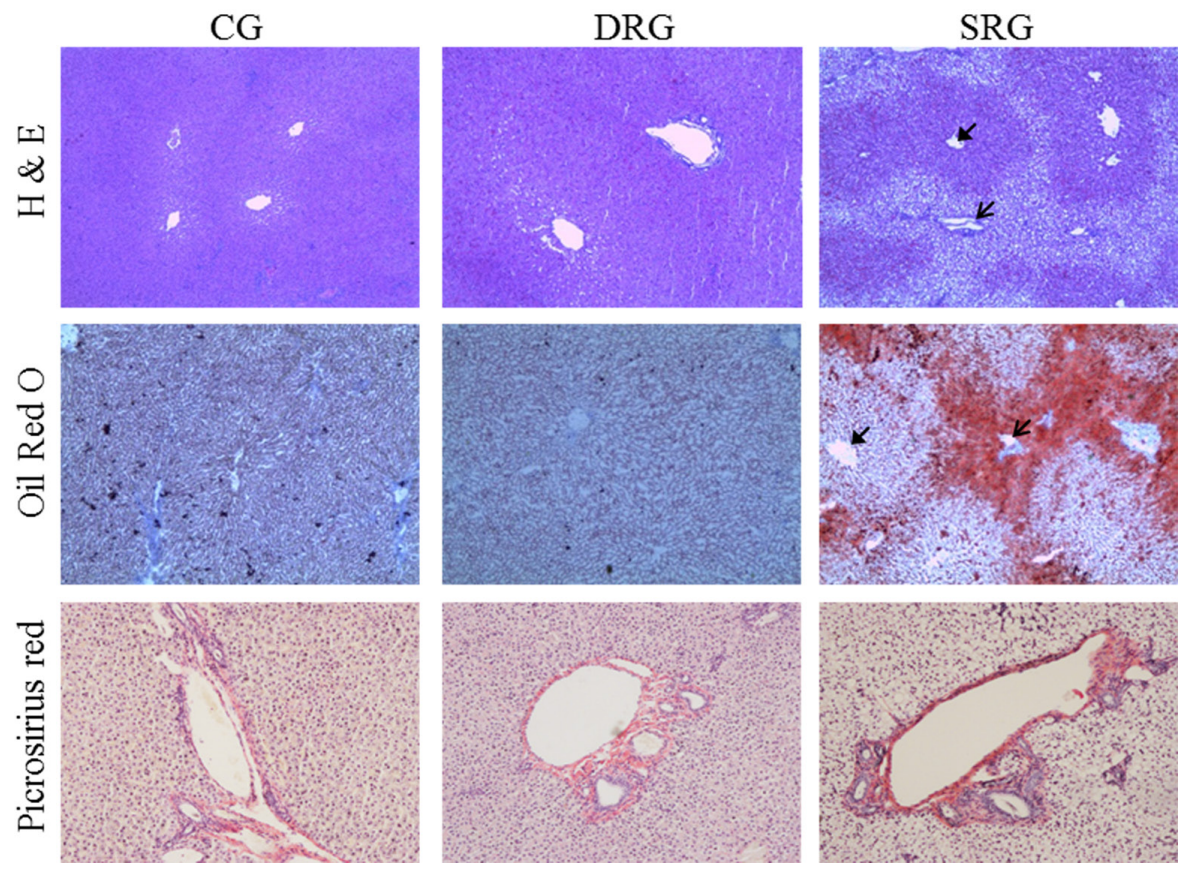

Figure 6. Representative liver images of $\mathrm{H} \& \mathrm{E}$, Oil Red $\mathrm{O}$, and picrosirius red staining in CG, NRG, and SRG sheep. H \& E and oil Red O staining images (40× magnification), picrosirius red staining images (100× magnification). Thin arrow, liver portal area; thick arrow, hepatic lobules.

Table 1. Analysis of biochemical parameters in the bile and liver.

\begin{tabular}{cccc}
\hline & CG $(\mathrm{n}=4)$ & NRG $(\mathrm{n}=4)$ & SRG $(\mathrm{n}=6)$ \\
\hline Bile & & \\
\hline TC $(\mathrm{mmol} / \mathrm{L})$ & $0.45 \pm 0.06$ & $0.27 \pm 0.12$ & $0.43 \pm 0.12$ \\
TBA $(\mu \mathrm{mol} / \mathrm{L})$ & $304.8 \pm 2.3$ & $307.8 \pm 1.2$ & $318.0 \pm 8.8$ \\
\hline Liver & & \\
\hline Glycogen $(\mathrm{mg} / \mathrm{g})$ & $4.41 \pm 2.52$ & $2.70 \pm 0.81$ & $2.81 \pm 0.48$ \\
TC $(\mu \mathrm{mol} / \mathrm{g})$ & $7.6 \pm 3.42^{\mathrm{a}}$ & $21.58 \pm 2.31^{\mathrm{b}}$ & $14.08 \pm 3.79^{\mathrm{c}}$ \\
\hline
\end{tabular}

Values are presented as the mean $\pm \mathrm{SD}$; values within a row that do not share a common superscript are significantly different $(P<0.05)$. 


\section{DISCUSSION}

Based on the characteristics of NEB [1], a model of FLD in lactating thin-tail sheep was developed by restricted feeding. With restriction feeding, serum glucose concentrations were sharply decreased in SRG sheep compared to controls that was due to the large energy requirements for lactation and low energy provided by a restricted diet. SRG sheep showed the lowest RQUICKI index value indicated decreased insulin sensitivity [9]. Serious NEB and LFCs were possibly caused by decreased insulin sensitivity of the liver, which led to inhibition of glucose utilization $[8,4]$. In this study, lactating sheep required high glucose precursors intake to maintain milk production. While non-lactating NRG sheep demonstrated lower fat mobilization, which was considered to contribute to the high concentrations of serum glucose, as compared to SRG sheep [6]. Under normal conditions, acetylcoenzyme A, a metabolite of NEFA, can be completely oxidized by oxaloacetic acid to produce adenosine triphosphate. Meanwhile, in a state of NEB, oxaloacetic acid, which is generated by glycolysis and glycogenic amino acids, tends to be used for gluconeogenesis [11]. However, a generous amount of NEFA is incompletely oxidized to generate ketone body, which is a major component of BHBA. With restricted feeding, an increase in serum NEFA among NRG sheep was also detected during the early stage of lactation, but serum glucose remained relatively high without milking, indicating complete oxidation of NEFA.

In the liver, some NEFA is esterified to generate triglycerides, phosphatides, and cholesterol ester. These three metabolites are then discharged from the liver to the blood in the form of very low density lipoprotein (VLDL). In the blood, following the dissociation of triglyceride, VLDL is gradually metabolized to LDL-C [14]. As shown in Table 1, compared with controls, liver TC concentrations were significantly higher in NRG sheep than those in the SRG sheep, while liver triglyceride were significantly lower. As a result, an abnormal secretion of liver TC in SRG sheep was considered. The high level of liver TC in NRG sheep was considered to induce removal of TGs from the liver in the form of VLDL. Compared with CG sheep, although higher levels of liver TC were detected in SRG sheep on postpartum day 16, these levels were considered too low to induce significant depletion of triglycerides from the liver. In consider- ation of the biochemical parameters in SRG sheep, high serum NEFA concentrations generate high levels of BHBA and TGs in the liver, but compared with NRG sheep, low concentrations of TC in SRG sheep were detected in the liver and serum, which we considered as a contributing factor of triglyceride accumulation in the livers of SRG sheep. This status is considered unfavorable for TC synthesis in a state of NEB because of energy requirements for TC synthesis.

In this study, the increase in serum AST and LDH was considered to cause by oxidative stress in mitochondria $[15,16]$. Dysregulation of serum AST and LDH is considered a characteristic of FLD in dairy cows. While in this study, LFC was elevated on postpartum day 4, but a significant increase in serum LDH was detected on postpartum day 13. Thus, it appears that high serum AST and LDH concentrations signify prolonged periods of LFC, and LDH concentrations was considered more sensitively than AST for LFC caused by NEB. In this study, liver pathological characteristics showed that FLD caused by NEB had a major impact on LFC, although no significant liver fibrosis was detected. While different from FLD caused by high-fat diet, TG was first accumulates around the hepatic lobules and LFC of NEB sheep was first detected around the liver portal area $[2,19]$. The venous blood in the hepatic lobules had low oxygenation concentrations which conveyed a weak oxidizing effect on fat that usually leads to accumulation of triglyceride by administration of a high fat diet. The liver portal area is composed of the hepatic artery, portal vein, and bile duct. Peripheral blood, which contains abundant nutrients and oxygen, is preferably metabolized at the liver portal area, and the metabolites are then transported to the hepatic lobule or discharged through the bile duct. In the NEB state, high concentrations of NEFA are prioritized for oxygenation in the liver portal area, which results in triglyceride accumulation. However, to clarify this pathological characteristics caused by LFC in the portal area will require further research.

\section{CONCLUSIONS}

FLD of sheep was induced by restricted feeding and lactation administration. Sheep accumulated significant LFC by postpartum day 4 and severe LFC was detected 10-13 days after initiation of the restricted diet. SRG sheep showed a severe NEB that significant 
low levels of serum glucose and high levels of NEFA and BHBA were detected, but blood biochemical parameters were questionable for evaluating the severity of LFC, and a high level serum LDH and AST concentrations signify prolonged LFC accumulation.

\section{MANUFACTURERS}

${ }^{1}$ Cone Instruments, LLC. Cleveland, OH, USA.

${ }^{2}$ Beijing Jiuqiang Biotechnology Co., Ltd. Beijing, China.

${ }^{3}$ Beckman Coulter Inc. Brea, CA, USA.

${ }^{4}$ Nanjing Jiancheng Biotechnology Institute Co., Ltd. Nanjing, China.
Acknowledgements. This work was supported by the National Nature Science Foundation of China (31502133), National Program on Key Research Project of China (2016YFD0501206), Post-doctoral Foundation in Heilongjiang Bayi Agricultural University (LRB15-18).

Ethical approval. The study protocol was approved by the Committee on the Ethics of Animal Experiments of the Heilongjiang Bayi Agricultural University (Permit Number: 20120319-1).

Declaration of interest. The authors report no conflicts of interest. The authors alone are responsible for the content and writing of the paper.

\section{REFERENCES}

1 Allen M.S., Bradford B.J. \& Harvatine K.J. 2005. The cow as a model to study food intake regulation. Annual Review of Nutrition. 25: 523-547.

2 Bedossa P. 2012. Lesions in nanolcoholic fatty liver disease. La Revue du praticien. 62: 14-19.

3 Bobe G., Amin V.R., Hippen A.R., She P., Young J.W. \& Beitz D.C. 2008. Non-invasive detection of fatty liver in dairy cows by digital analyses of hepatic ultrasonograms. Journal of Dairy Research.75: 84-89.

4 Chalmeh, A., Pourjafar M., Nazifi S., Momenifar F. \& Mohamadi M. 2015. Insulin Resistance in Different Physiological States of High Producing Holstein Dairy Cows. Acta Scientiae Veterinariae. 43:1255.

5 Civelek T., Aydin I., Cingi C.C., Yilmaz O. \& Kabu M. 2011. Serum non-esterified fatty acids and beta-hydroxybutyrate in dairy cows with retained placenta. Pakistan Veterinary Journal. 31: 341-4.

6 Dann H.M., Morin D.E., Bollero G.A., Murphy M.R. \& Drackley J.K. 2005. Prepartum intake, postpartum induction of ketosis, and periparturient disorders affect the metabolic status of dairy cows. Journal of Dairy Science. 88: 3249-3264.

7 Erinoso H.O., Hoare S. \& Weaver L.T. 1992. Is cow's milk suitable for the dietary supplementation of rural Gambian children? 2. Patterns of cow's milk intake. Annals of Tropical Paediatrics. 12: 367-373.

8 Holtenius P. \& Holtenius K. 1996. New aspects of ketone bodies in energy metabolism of dairy cows: a review. Zentralblatt fur Veterinarmedizin Reihe A. 43: 579-587.

9 Holtenius P. \& Holtenius K. 2007. A model to estimate insulin sensitivity in dairy cows. Acta Veterinaria Scandinavica. 49: 29.

10 Kelton D.F., Lissemore K.D. \& Martin R.E. 1998. Recommendations for recording and calculating the incidence of selected clinical diseases of dairy cattle. Journal of Dairy Science. 81: 2502-2509.

11 Lucy M.C., Escalante R.C., Keisler D.H., Lamberson W.R. \& Mathew D.J. 2013. Glucose infusion into early postpartum cows defines an upper physiological set point for blood glucose and causes rapid and reversible changes in blood hormones and metabolites. Journal of Dairy Science. 96: 5762-5768.

12 Miksa I.R., Buckley C.L. \& Poppenga R.H. 2004. Detection of nonesterified (free) fatty acids in bovine serum: comparative evaluation of two methods. Journal of Veterinary Diagnostic Investigation. 16: 39-44.

13 Rector R.S., Thyfault J.P., Uptergrove G.M., Morris E.M., Naples S.P., Borengasser S.J., Mikus C.R., Laye M.J., Laughlin M.H., Booth F.W. \& Ibdah J.A. 2010. Mitochondrial dysfunction precedes insulin resistance and hepatic steatosis and contributes to the natural history of non-alcoholic fatty liver disease in an obese rodent model. Journal of Hepatology. 52: 727-736.

14 Sejersen H., Sorensen M.T., Larsen T., Bendixen E. \& Ingvartsen K.L. 2012. Liver protein expression in dairy cows with high liver triglycerides in early lactation. Journal of Dairy Science. 95: 2409-2421.

15 Shi X.X., Li D.D., Deng Q.H., Li Y., Sun G.Q., Yuan X., Song Y.X., Wang Z., Li, X.B., Li X.W. \& Liu G.W. 2015. NEFAs activate the oxidative stress-mediated NF-kappaB signaling pathway to induce inflammatory response in calf hepatocytes. Journal of Steroid Biochemistry. 145: 103-112.

16 Song Y., Li N., Gu J., Fu S., Peng Z., Zhao C., Zhang Y., Li X., Wang Z., Li X. \& Liu G. 2016. $\beta$-Hydroxybutyrate induces bovine hepatocyte apoptosis via an ROS-p38 signaling pathway. Journal of Dairy Science. 99: 9184-9198 
17 Xu C., Sun L.W., Xia C., Zhang H.Y., Zheng J.S. \& Wang J.S. 2016. H-Nuclear magnetic resonance-based plasma metabolic profiling of dairy cows with fatty liver. Asian-Australasian Journal of Animal Sciences. 29: $219-229$.

18 Xu C., Xu Q.S., Chen Y.Y., Yang W., Xia C., Yu H.J., Zhu K.L., Shen T.Y. \& Zhang Z.Y. 2015. The relationship between Fibroblast Growth Factor-21 and characteristic parameters related to energy balance in dairy cows. BMC Veterinary Research. 271: 1-7.

19 Yang W., Wu D.J., Zhang R.H., Deng Q.H., Ding H.Y., Tian Y., Zhang M., Liu G.W., Wang Z., Li X.B. \& Li X.W. 2015. Effects of Propylthiouracil-Induced Hypothyroidism on Nonalcoholic Fatty Liver Disease. Pakistan Veterinary Journal. 35: 1-6. 